\title{
Optimising treatment of CF pulmonary exacerbation: a tough nut to crack
}

\author{
Sonya L Heltshe, ${ }^{1,2}$ Christopher H Goss ${ }^{1,2,3}$
}

Pulmonary exacerbations (PExs) are a common occurrence in cystic fibrosis (CF) and their impact real, detrimentally affecting quality of life, ${ }^{12}$ morbidity ${ }^{3} 4$ and mortality. ${ }^{5}$ Treatment often includes a prolonged course of intravenous aminoglycosides, beta-lactams and increased airway clearance but there is neither consensus nor standard protocol for therapeutic options in the setting of CF PEx. The United States CF Foundation conducted a systematic review and published guidelines $^{67}$ for the treatment of a CF PEx; the data presented in the systematic review highlighted the paucity of evidence that guides current clinical management. Recent advances in CF chronic therapies such as inhaled antibiotics, cystic fibrosis transmembrane conductance regulator (CFTR) modulators, mucolytics and macrolides have proven to reduce the occurrence of PEx or delay the time to next exacerbation; however, very limited research ${ }^{8}$ has occurred in the area of acute management of PEx, including the development of novel therapeutics. This is due, in part, to the challenges in conducting such studies. Randomised, controlled trials of acute PEx management are mired by challenges, including variability in treatment location (home vs inpatient vs both), duration and selection of antibiotics and use of mucolytics or corticosteroids-many of which are driven by differences in physician goals or patient preferences. Conducting a trial in an environment with this constellation of variable treatment factors can be a daunting task, but without such trials, we can never improve the care of acute PEx for our patients with CF.

Dentice $e t a l^{9}$ report findings of a randomised, blinded, placebo controlled trial

\footnotetext{
${ }^{1}$ Department of Pediatrics, University of Washington School of Medicine, Seattle, Washington, USA; ${ }^{2}$ Cystic Fibrosis Foundation Therapeutics Development Network Coordinating Center, Seattle Children's Research Institute, Seattle, Washington, USA; ${ }^{3}$ Division of Pulmonary and Critical Care Medicine, Department of Medicine, University of Washington School of Medicine Seattle, Seattle, Washington, USA
}

Correspondence to Dr Christopher H Goss, Professor of Medicine and Pediatrics, University of Washington Medical Center, Campus Box 356522, 1959 N.E. Pacific, Seattle, WA 98195, USA; goss@u.washington. edu

of hypertonic saline (HS) administered during a hospital admission for PEx in CF adults. HS is an osmotic agent responsible for hydrating airway surface liquid and improving mucus clearance in the CF lung and is commonly used as a chronic therapy $(65.7 \%(n=18840)$ of US patients over 6 years old in 2014). ${ }^{10}$ The primary objective of the study was to show that HS was harmful or beneficial when used as an adjunctive therapy during a PEx based on tolerability, length of hospital stay, rate of resolution of signs and symptoms of acute PEx and time to next exacerbation. While the primary endpoint was not met (HS group hospital stay was 1 day shorter than placebo with 95\% CI 0 to 2 days), the authors were able to show astoundingly high adherence and tolerability to HS (albeit in an inpatient setting) in addition to significant improvements in congestion symptoms and recovery of pre-exacerbation lung function by discharge $(75 \%$ vs $57 \%)$. The authors should be commended for conducting this relatively large study of CF exacerbation $(n=132)$. While they were unable to show a significant reduction in hospital days, they did see indications of improved outcomes with HS in spite of the reduced duration of treatment (only 1 day shorter).

While the paper demonstrates strong data that symptoms as measured by a Leikert scale and lung function as measured by $\mathrm{FEV}_{1}$ were improved, length of hospital stay as an outcome measure for CF PEx studies raises some interesting challenges. Length of hospital stay is a highly meaningful measure of clinical efficacy in that it reduces patient burden and health system costs and should be a direct reflection of clinical improvement such as $\mathrm{FEV}_{1}$ recovery and symptom resolution. However, the authors do not describe what constituted criteria for discharge and instead state that discharge timing remained at the discretion of the treating physician. How much improvement in $\mathrm{FEV}_{1}$ or symptoms was enough to warrant discharge? While the study was blinded with a masked placebo using $0.19 \%$ saline (a clear strength of the study), having clear criteria is essential to understanding the meaning of their primary endpoint. The best chance for moving the needle on an outcome such as length of stay is to provide specific criteria directing intravenous discontinuation or patient discharge based on specific lung function thresholds and/or symptom resolution. Doing so would ensure treatment duration be a direct measure of patient improvement, less subject to physician and patient interpretation, decision and variability. In a recent study of PEx, 46\% $(n=102)$ of physicians reported intent to treat for a fixed duration (commonly 14 days) ${ }^{10} 11$ and only add hospital or intravenous days if a patient is not responding to therapy. Physicians and patients may be reluctant to shorten an intravenous course of antibiotics unless they note a marked improvement in patient signs and symptoms.

The advantage of using PEx treatment duration to measure efficacy in a controlled trial is to remove it as a confounding factor for any other clinical measures otherwise chosen to demonstrate effectiveness. Standardising treatment discontinuation criteria would objectify the endpoint; however, implementing a protocol to dictate treatment length is not without challenges. What would be universally acceptable criteria for patient discharge or extending the treatment duration? A $10 \%$ improvement in $\mathrm{FEV}_{1}$ plus significant reductions in chest congestion might be a meaningful benchmark for one patient, whereas another, with more advanced disease, may never be expected to meet that threshold. Would it be acceptable to let the former patient discontinue PEx therapy after placing a peripherally inserted central catheter and only 4 days of therapy (if that is when they met the a priori definition) while the latter languishes on in the hospital for weeks with an $8 \% \mathrm{FEV}_{1}$ improvement? The unique patient profiles, therapeutic needs and achievable outcomes make research in the area of CF PEx extremely difficult. An alternative to using duration as an outcome would be to first optimise exacerbation treatment, and then fix the antibiotic period in subsequent studies of adjunct therapies to eliminate its confounding effect on clinically relevant measures such as lung function or symptom resolution.

What other key endpoints should one consider in PEx studies? Time to next acute PEx was reported in this study by Dentice $e t a l^{9}$ and others; ${ }^{12} 13$ a number of studies have suggested that this is not a relevant endpoint. ${ }^{14} 15$ When one evaluates the time to next PEx for an acute treatment, there is a clear disconnect between the primary event and the next event. 
Exacerbations are likely stochastic events that relate more to the timing of viral upper respiratory tract infections and adherence to routine treatments. Treatment failure is also a possible endpoint, but patient response to antibiotics for exacerbation is fairly consistent; thus, rates of true treatment failure (like retreatment in 30 days) are likely rare. ${ }^{16}$ The key endpoints will need to resonate with patients and providers. These endpoints will need to include lung function and symptoms preferably assessed using a measure like the CF Respiratory Symptom Diary to clearly track treatment response. ${ }^{17}$

The study by Dentice $e t$ al is not unlike other interventional trials ${ }^{18-22}$ in CF PEx - unable to conclusively demonstrate a clinically meaningful treatment effect despite promising trends in a study population that was too small to show efficacy. HS appears to be tolerated and while it did not significantly reduce length of hospital stay, it is probably safe to say it does not lengthen it, while showing some evidence that recovery of pre-PEx $\mathrm{FEV}_{1}$ is improved with HS. Is this enough evidence to advocate for its adoption in overall management of PEx? Maybe the more important question: are we putting forth our best effort to advance the care for patients with CF during their most vulnerable periods of acute illness? Yes, it is expensive to conduct very large trials for conclusive results. Yes, it is hard to impress upon physicians the need for equipoise when standardising aspects of care long thought to be the 'art of medicine'. Yes, it is difficult to educate patients and families on the importance of participating in research even when they are very sick. But when it comes to CF PEx we are not currently practising evidencebased medicine, and decades with dozens of inconclusive studies are not getting us much closer. We are in an exciting era where CFTR modulators are available for almost half the CF population; it is about time we start devoting resources to appropriately address PEx.

Contributors Drafting the manuscript for important intellectual content: all authors.

Funding $\mathrm{CHG}$ receives funding from the Cystic Fibrosis Foundation, the NIH (RO1HL103965, R01HL113382, R01Al101307, U M1HL119073, P30DK089507) and the FDA (R01FD003704). SLH receives funding from the Cystic Fibrosis Foundation and the NIH (P30DK089507, R01DK095738, UM1HL119073, R01DK095869).

Competing interests None declared.

Provenance and peer review Commissioned; externally peer reviewed.

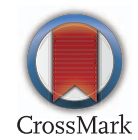

To cite Heltshe SL, Goss CH. Thorax 2016;71:101102.

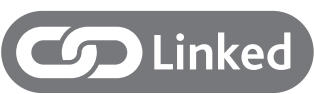

- http://dx.doi.org/10.1136/thoraxjnl-2014-206716

Thorax 2016:71:101-102.

doi:10.1136/thoraxjnl-2015-208057

\section{REFERENCES}

1 Britto MT, Kotagal UR, Hornung RW, et al. Impact of recent pulmonary exacerbations on quality of life in patients with cystic fibrosis. Chest 2002;121:64-72.

2 Habib AR, Manji J, Wilcox PG, et al. A systematic review of factors associated with health-related quality of life in adolescents and adults with cystic fibrosis. Ann Am Thorac Soc 2015;12:420-8.

3 de Boer K, Vandemheen KL, Tullis E, et al. Exacerbation frequency and clinical outcomes in adult patients with cystic fibrosis. Thorax 2011;66:680-5.

4 Lieu TA, Ray GT, Farmer G, et al. The cost of medical care for patients with cystic fibrosis in a health maintenance organization. Pediatrics 1999;103:e72.

5 Liou TG, Adler FR, Fitzsimmons SC, et al. Predictive 5-year survivorship model of cystic fibrosis. Am J Epidemiol 2001;153:345-52.

6 Flume PA, Mogayzel PJ Jr, Robinson KA, et al. Cystic fibrosis pulmonary guidelines: treatment of pulmonary exacerbations. Am I Respir Crit Care Med 2009;180:802-8.

7 Stenbit AE, Flume PA. Pulmonary exacerbations in cystic fibrosis. Curr Opin Pulm Med 2011;17:442-7.
8 Hurley MN, Prayle AP, Flume P. Intravenous antibiotics for pulmonary exacerbations in people with cystic fibrosis. Cochrane Database Syst Rev 2015;7:CD009730.

9 Dentice RL, Elkins MR, Middleton PG, et al. A randomised trial of hypertonic saline during hospitalisation for exacerbation of cystic fibrosis. Thorax 2016;71:141-7.

10 Cystic Fibrosis Foundation Annual Data Report to the Center Directors. https://www.cff.org/2014_CFF_ Annual_Data_Report_to_the_Center_Directors.pdf/

11 West NE. NACFC symposium presentation: The STOP trial: an observational pilot and feasibility study of exacerbation management. Pediatr Pulmonol 2015; S41:124-6.

12 Sequeiros IM, Jarad N. Factors associated with a shorter time until the next pulmonary exacerbation in adult patients with cystic fibrosis. Chron Respir Dis 2012;9:9-16.

13 Smith AL, Doershuk C, Goldmann D, et al. Comparison of a beta-lactam alone versus beta-lactam and an aminoglycoside for pulmonary exacerbation in cystic fibrosis. J Pediatr 1999;134:413-21.

14 Heltshe SL, Goss CH, Thompson V, et al. Short-term and long-term response to pulmonary exacerbation treatment in cystic fibrosis. Thorax. Published Online First: $24 \mathrm{Apr}$ 2015. doi:10.1136/thoraxinl-2014-206750.

15 Waters V, Stanojevic S, Klingel M, et al. Prolongation of antibiotic treatment for cystic fibrosis pulmonary exacerbations. J Cyst Fibros 2015;14:770-6.

16 Parkins MD, Rendall JC, Elborn JS. Incidence and risk factors for pulmonary exacerbation treatment failures in patients with cystic fibrosis chronically infected with Pseudomonas aeruginosa. Chest 2012;141:485-93.

17 Goss CH, Edwards TC, Ramsey BW, et al. Patient-reported respiratory symptoms in cystic fibrosis. J Cyst Fibros 2009;8:245-52.

18 Bakker EM, Volpi S, Salonini E, et al. Small airway deposition of dornase alfa during exacerbations in cystic fibrosis; a randomized controlled clinical trial. Pediatr Pulmonol 2014;49:154-61.

19 Dovey M, Aitken ML, Emerson J, et al. Oral corticosteroid therapy in cystic fibrosis patients hospitalized for pulmonary exacerbation: a pilot study. Chest 2007;132:1212-18

20 Dwyer TJ, Robbins L, Kelly P, et al. Non-invasive ventilation used as an adjunct to airway clearance treatments improves lung function during an acute exacerbation of cystic fibrosis: a randomised trial. J Physiother 2015;61:142-7.

21 Grossmann RE, Zughaier SM, Kumari M, et al. Pilot study of vitamin D supplementation in adults with cystic fibrosis pulmonary exacerbation: a randomized, controlled trial. Dermatoendocrinol 2012;4:191-7.

22 Yau YC, Ratjen F, Tullis E, et al. Randomized controlled trial of biofilm antimicrobial susceptibility testing in cystic fibrosis patients. I Cyst Fibros 2015;14:262-6. 